das in manchen Fällen vom Brutgeschäft beeinflusst sein kann, zu ändern. Jedenfalls hoffe ich nach der Rückkehr von der Südpolarexpedition einen ausführlichen Beitrag zur geographischen Verbreitung der Meeresvögel insbesondere jener der sũdlichen Breiteu liefern zu können.

\title{
Zur Ornis des Thales der Drewenz
}

(im Weichbilde der Stadt Neumark i. Wpr.).

\section{Von Fritz Braun.}

Mag das Wanderleben eines westpreussischen Schulamtskandidaten auch manche Unannehmlichkeiten mit sich bringen, der Freund der heimischen. Ornis wird diese gern in den Kauf nehmen, bringt ihm doch grade der unstäte Wandel eines modernen Bildungsreisenden grosse, nicht zu unterschätzende Vorteile. Vor anderen lernt er erkennen, dass unser Vaterland auch auf kleinerem Raum durchaus kein einheitliches Gebiet ist, dass dem Forscher in scheinbar recht ähnlichen Gegenden eine gar verschiedene Ornis entgegentritt - eine beherzigenswerte Warnung vor vorschnellem Verallgemeinern. Er begreift, wie wenig damit gesagt ist, eine Vogelart kommt bis hier und dahin vor, er sieht ein, dass die einzelnen Arten inmitten ihres Gebietes hier dichter, dort dünner siedeln und grossen Landstrecken inmitten ihrer Verbreitungszone sogar völlig fehlen, ohne dass man für ihr Verschwinden sogleich einen nahe liegenden Grund anführen könnte. Die Feststellung der verschiedenen Siedelungsdichte einer und derselben Art wird für die genaue Schilderung ihrer Lebensweise, für die Aufklärung der geheimen Zusammenhänge zwischen dem Leben grade dieses Vogels mit der Bodenform, der Vegetation und der Wirtschaftsgeographie oft viel, viel wichtiger sein, als die zufällige Notierung eines vorschnellen Pioniers, der über das Reich seiner Artgenossen hinausstrebte.

Wie angenchm wäre es für uns, wenn wir z. B. für Fringilla serinus eine Karte seiner Siedelungen und seiner Siedelungsdichte besässen. Damit wäre für die kausale Erklärung seiner Nordwanderung wohl die wichtigste Vorarbeit geleistet.

Durch solche Arbeiten kann wegen der Gründlichkeit, die sie verlangen, auch nicht so leicht gesündigt werden, wie bei der isolierten Angabe eines neuen Nistplatzes. In einem der letzten Jahrgänge der "gefiederten Welt" las ich zu meinem Schrecken 
die Notiz, dass der Karmingimpel (Loxia erythrina) bei Zoppot ein häufiger Brutvogel sei, wovon bei uns in Westpreussen kein Mensch etwas weiss. Sicher ein Fall, wo ein fromder Badegast erfuhr, dass dort irgendwann einmal brittende Karmingimpel gefunden seien und nun schlankweg darauflos dekretierte: „Bei Zoppot brätet Loxia erythrina." Der Deutsche im Reich, der dann das Zeug liest, glaubt daraufhin wohl, bei uns dürfe man nur in den Wald gehen, um dem Flötensange dieses nordischen Gimpels zu lauschen.

Im Folgenden wollen wir uns mit dem Thale der Drewenz beschäftigen, dem Thale des südlichsten Nebenflusses, der auf preussischer Erde der mächtigen Weichsel von rechts her zuströmt.

In diesem Thale liegt auch die Schulstadt Neumark, der Sitz des preussischen Landratamts Löbau. Dieser Ort ist eine charakteristische Passstadt. Wollte man das Thal der Drewenz überschreiten, so bietet sich hier die letzte Gelegenheit, denn nordwärts treten die Hänge der Randberge weiter auseinander und schauen auf breite, sumpfige Wiesen herab, die dem Wanderer zur Zeit des Hochwassers und der Schneeschmelze unüberwindliche Hindernisse bereiten, und südwärts treffen wir tiefgründiges Moor.

Durch Wiesen und Felder, Torfbrüche und Hopfenplantagen und Kartoffeläcker strömt die Drewenz mit schmalem, tiefem, strudelreichem Wasserlauf, von dem sich nur hier und da schmale Altwasser abzweigen, in denen geiler Schachtelbalm, selten speerschäftiges Rohr emporstrebt. Je nach dem Gange der Serpentinen ist das Ufer abwechselnd steil und flach, die Steilhänge selten höber als vier bis fünf Fuss, der gelbe Strand nur selten breiter als einen knappen Meter. Die Breite des ganzen Erosionsthales, das der Fluss sich im Laufe der Jahrtausende schuf, betrïgt durchschnittlich $1100 \mathrm{~m}$., Wege und Siedelungen folgen zumeist rechts und links dem Hange der Randberge.

Ursprünglich bedeckte diese Höhen rauschender Wald. Aber Schlag um Schlag fielen die Forsten der Axt zum Opfer. Wald ist Geld und unausgegebenes Geld lässt den Enkel der Schlachzizen nicht schlafen. Jetzt sind die Höhen von Kaluga bis Brattian kahl und öde. In den Schluchten kummern zwar noch hier und da kleine Bestände, aber nur die königliche Forst Kosten sendet ihre Vorposten bis an das Ufer der Drewenz herab. 
Sogar die wenigen Bäume, die z. B. nördlich von Neumark die Drewenz begleiten, dünnzweigige, sperrig belaubte Weiden und Pappeln, sind nicht grade geeignet, gefiederte Bewohner anzulocken. $\mathrm{Zu}$ alle dem sind auch die weidenbestandenen Ufergelände, die bei grösseren Flüssen, z. B. der Weichsel, eine Fülle insektenfressender Vögel beherbergen, an der Drewenz nur selten und klein.

Der durch Vernichtung der Wälder bewirkte Ilolzmangel zwang den Bauern, auch die Einzelbäume und das Gesträuch arg mitzunehmen, um Herdholz und Heizmaterial zu gewinnen. So sehen wir denn an den Hütten der liäthner oft Berge von Kiefern und Weidengeäst aufgeschichtet, trägt fast jeder Baum Spuren der unbarmherzigen Axt, die den misshandelten Bäumen Zweig um Zweig geraubt, selbstverschuldeter Not gehorchend.

Dieser kargen, sonnenhellen Landschaft, reich an Sand und Moor, arm an Fruchtbarkeit, Behagen und schattendem Baumwuchs entspricht auch die Ornis. Jedoch milssen wir zugeben, dass diese trotz der Einförmigkeit der Boden- und Vegetationsform eine überaus reiche ist, dass der landfremde Ankömmling fast tagtäglich durch eine neue Beobachtung angenehm enttäuscht wird, damit seine Erwartungen steigert und sich in ihnen selten getäuscht findet.

Wandern wir auf sandigem Pfade längs der Höhe dahin, so finden wir rechts und links die Vögel der Kultursteppe, der Kultursteppe in ibrer sandigen, unfruchtbaren Form.

Auffallend war es mir, dass die verschiedenen Ammerarten, die weiter im Norden, in den Werdern der Weichsel und ihren Grenzgebieten sich gegenseitig zu.verdrängen scheinen, hier friedlich mit- und nebeneinander hausen. Bei Kaluga erschallten mir aus dem geräumigen Wipfel einer und derselben Kopfweide die Lieder von Emberisa miliaria, E. citrinella und hortulana entgegen und citrinella und miliaria fand ich recht häufig beisammen.

Bei weitem am meisten findet man $E$. miliaria. Sie ist der Charaktervogel des Thales. Auf jeder dritten Weide, auf jeder boczemecka, wie der Pole die Bildstöckel am Kreuzweg nennt, spinnt sie ihren Leiersang. Nahm ich das Tesching zur Hand und stellte ich der unschönen Base des Goldammers mit den Schroten nach, so war die Beute stets reichlich genug. Selbst mitten im Felde, fernab von Strauch und Stein, wirkt sie ihr eintöniges, ermüdendes Tongewebe. 
Wegen ihrer erstaunlichen Häufigkeit wäre diese Ammer hier ganz geeignet, ein jagdbares Wild abzugeben. Bei ihrer verhältnismässigen Grösse lohnt sie schon den Schrotschuss der leichten Büchse. Eine Ausrottung der Art wäre kaum zu befürchten, dagegen nur wünschenswert, dass in die durch Pulver und Blei geschaffenen Lücken ihres Bestandes die schönfarbige Goldammer, der hellstimmige Ortolan einrücken.

Sitzen die Grauammern hoch oben in den Weidenkronen, so erkennt man sie - ganz abgesehen von der Färbung und dem schrillen, unverkennbaren Getön - schon an der balanzierenden Haltung des Körpers, die den Schwanz selten zur Ruhe kommen lässt. Ihre unmässig grossen Füsse sind nicht mehr recht geeignet, dem unförmlichen Vogel auf dem schwankenden Weidenästchen einen sicheren Sitz zu bereiten.

Nicht viel minder zahlreich als $\boldsymbol{E}$. miliaria ist $\boldsymbol{E}$. citrinella, die hier wie anderswo sich von allen Ammern noch am ehesten in das Waldleben findet. Ich fand sie mitten im Kosener Walde, sofern nur eine mässig grosse Lichtung den Ausflug ins Feld ermöglichte, und hörte sie mitten im Buchenwalde, 80-100 m. vom Lichten entfernt, ihren Singsang leiern.

Emberiza hortulana findet man am ehesten an der Weissenburg-Neumark-Strasburger Chaussee; auch sie ist hier viel häufiger als in der Danziger Gegend.

Neben den Ammern treiben die Lerchen ihr Wesen. Alauda cristata stolziert selbst auf dem Schulhofe einher, um dicht an der Thür nach einem verlorenen Brocken zu picken. 'Trotz ihrer grossen Zahl sind die Reviere von Alauda arvensis auch hier genau bestimmt, wie ich an einigen zerstreut siedelnden Bodensängern feststellen konnte, die ich immer wieder an denselben Punkten fand.

Von Finkenarten findet man in der Nähe des Städtchens Neumark, das mit seinen Gärten und Alleen ein Gebiet für sich bildet, zumeist den Grünfinken (Fringilla chloris), der auf engem Raum in Datzenden von Paaren siedelt und uns überall mit seinem Leiersange verfolgt. Bei dem stürmischen Wetter einiger Maitage hörten wir nur das ewig wiederholte „Schwunsch"; offenbar macht dieser Ruf dem Sänger dann weniger Mühe als die längeren, klirrenden Strophen. Bei solcher Siedelungsdichte der Grünfinken muss selbst die minnigliche Streitlust zurücktreten, teilen drei, vier Männchen mitunter friedlich denselben Sitz, 
Häufig genug ist an dem gleichen Ort auch der Stieglitz (Fringilla carduelis), den man oftmals von einem freien Zweig der halbwüchsigen Gartenfichten, die der Grünfink fast ängstlich meidet, seine kecke Weise zwitschern hört. Der Stieglitz ist der schönste Schmuckvogel der Neumärker Gärten; der IIänfling (Fringilla cannabina) streicht dagegen mehr in den Feldern umher. Noch in der zweiten Maiwoche sah ich die schmucken Vögel, Männchen und Weibchen in Fltigen miteinander, ohne Hader und Zank, gesellig das Land durchstreifen. Fbenso verhielt sich auch der Stieglitz; noch am 16. Mai sah ich ihn in Flügen von 4 bis $6 \mathrm{Köpfen,} \mathrm{also} \mathrm{noch} \mathrm{nicht} \mathrm{in} \mathrm{paarweiser} \mathrm{Ab-}$ sonderung.

Eine besondere Vorliebe hat der Hünfling für die Holzlager der grossen Schneidemühlen, wo fast immer einige Hänflinge auf den ragenden Holzstapeln ihre flötenden Strophen singen, trotzdem kein grüner Baum zwischen den leuchtenden Dielen wächst.

Der Buchfink (Fringilla coelebs) ist in dem baumarmen Lande verhältnismässig selten und arm an Stimme, bänzlich feblen Zeisig (Fringilla spinus) und Girlitz (Fringilla serinus), der Charaktervogel der Gärten im Norden Danzigs. Für den Girlitz ist hier in der kahlen Kultursteppe kein Platz und die, zumeist noch dazu recht jungen Gärten des Städtchens Neumark sind doch eine gar zu bescheidene Oase in der baumlosen Wüste, um von fernher heikle Ansiedler anzulocken. So wird wohl noch manches Jahr vergehen, ehe sich hier der schwirrende Sang des Girlitz in die Strophen der Grünlinge mischt.

Vielleicht wird ein weiteres Anwachsen der Linden an unsern neuen Chausseen die Verbreitung der Girlitze befördern; in der Danziger Gegend hat der gelbgrüne Fink eine entschiedene Vorliebe für die Nachbarschaft alter, breitkroniger Linden und ähnlicher Laubbäume, denen zu Liebe er sogar auf die Nähe des Waldrandes verzichtet.

Auffällig ist im Drewenzthal das Fehlen von Star (Sturnus vulgaris) und Elster (Corvus pica). Die einheimischen wollen zwar des Fehlen des Stars bestreiten; da ich aber auf meinen tagtäglichen Wanderungen auch keinen einzigen Starmatz erschaute, der doch sonst nicht grade zu den. schwer zu erspähenden Geschöpfen zählt, blieb der Mangel immerhin auffällig genug, zumal wenn man aus den Starendorado der Werder kommt. Beiläufig: Wenn Friderich den Pirolpfiff ein Besitztum der Waldstare nennt, 
so irrt er; zu heuriger Osterzeit begleitete mich in dem waldarmen Danziger Werder der Pirolpfiff der Starmatze von Dorf zu Dorf.

Das Handwerk des Stars scheint hier ausschliesslich die Nebelkrïhe (Corvus cornix) zu üben, die mir durch ihr zutrauliches Wesen anfangs besonders auffiel. Lange Zeit lebte ich des Glaubens, auf dem Gute Weidenau gäbe es zahme Krähen. Bisher kannte ich nur das verschüchterte Krähenvolk in dem Weichbild der Grossstitdte, die in dem Menschen den Mörder fürchten, und diese Neumärker Kinder liessen mich getrost bis auf zwei, drei Schritt herankommen. Später erst wurde ich darüber belehrt, dass ich im Irrtum befangen eine Erscheinung als Ausnahme ansah, die hier die Regel bildet. Trotzdem sah ich die Nebelkrähen hier niemals zu so grossen Nistgesellschaften vereinigt wie weiter nordwärts, an dem Hange der pommerellischen Höhen.

Weil Dohle (Corvus monedula) und Elster fehlen und die Saatkrïhe (Corvus frugilegus) sich höchstens auf dem Strich und der Wanderung erspähen lässt, so ist Corvus cornix die einzige Vertreterin der Rabenvögel.

Dem Star ist das Drewenzthal wahrscheinlich zu baumarm und der Abhang der Randberge zu dürr. Zudem hat das treffliche Vorbild des seligen Lenz, die Starmätze durch Nistkästen anzulocken, bei den Neumärker Bürgern noch wenig Nachahmung gefunden.

Neben dem Hänfling treibt sich auf den Holzfeldern das Blaukehlchen (Lusciola cyanecula) umher. Der schönbrüstige Vogel scheint in unserem Gau recht innig an den Werken und dem Besitz des Menschen zu hängen. Im Danziger Werder findet man ihn viel seltener draussen in der Feldmark, in dem Weidengesträuch der Grabenränder, als in der Nähe der Wirtschaftsgebäude, zumal der Strohstaken, die durch Umfang und Höhe von dem Segen der Fruchtfelder Zeugnis geben.

Sein Verwandter, das Rotkehlchen (Lusciola rubecula) bewohnt nicht grade häufig das Unterholz der Neumärker Gärten, nachbarlich gesellt dem hellstimmigen Spötter (Sylvia hypolais), der Zaun- (Sylvia curruca) und Dorngrasmücke (Sylvia cinerea). Auch das Schwarzplättchen (Sylvia atricapilla) fand ich auf dem evangelischen Friedhof des Städtchens, dagegen nur äusserst selten die graue Gartengrasmücke (Sylvia hortensis), was auch wohl auf die Holzarmut der Gegend zurückgeführt werden muss. 
Sie alle findet man zumeist nur in Neumark selbst und in den Gärten der grössten Güter.

Nur die Dorngrasmücke trifft man auch in den zerstreuten Weidenbüschen der Drewenzniederung. Geht's gut, so kann man sie fünf, ja zehn Minuten lang auf ein bis zwei Meter Entfernung betrachten. Anstatt vor dem Beobachter zu fliehen, spielt der Vogel hier mit ihm Versteck und bleibt still und starr im Gezweige sitzen. Oftmals ist allerdings selbst in der geringsten Entfernung schwer, ihn zu entdecken, denn sein braungraues Kleid trägt Schutzfarben, die mit der Umgebung wunderbar übereinstimmen.

Û̉ber und zwischen diesen Laubbewohnern streifen die Meisen durchs Gezweig, vor allem Parus maior und Parus palustris in etwa gleicher Zahl. Von letzterer besucht ein Pärchen wohl tagtäglich den Schulgarten. Ich erkenne die beiden daran, dass dem schlanken Weibchen ein dicker, plustriger Gatte gesellt ist; vielleicht bedingt eine chronische Krankheit die plumpe Haltung des sonst so munteren Geschöpfs. Die beiden unzertrennlichen nisten wahrscheinlich auf einem dem Schulgarten benachbarten Friedbof und treffen auf ihrem täglichen Streifaug fast "pünktlich zur Sekunde" in den Baumkronen des Schulhofes ein. Blaumeisen (Parus coeruleus) sah ich bei Neumark so gut wie garnicht, so dass ich fast ihr Fehlen feststellen könnte.

Neben den Sumpfmeisen besuchen auch Trauerfliegenfänger (Muscicapa atricapilla) von Zeit zu Zeit den Scbulgarten. Im Tannengrün verborgen, konnte jch die weissbindigen Gesellen aus nächster Nähe beobachten. Längere Zeit gingen sie dicht vor meinen Augen ihrem rastlosen Gewerbe nach, bis sie mich schliesslich doch bemerkten und dann erschreckt von dannen stoben.

Den grauen Fliegenschnäpper (Muscicapa grisola), meinen guten Bekannten von Danzig her, der in vorigen Jahre in Gr. Walddorf hinter einer Dachrinne seine junge Brut aufzog, bekam ich in unserem Schulgarten gleichfalls zu sehen. Zumeist sehe ich das Pärchen auf der Nordostecke des Gymnasiums sitzen, um von dort unter lebhaftem tschrie-tschrie seine typischen Flugbogen $z u$ beschreiben. Hoffen wir, dass es an dem gastlichen Hause auch die Wiege seiner Kinder erbaut.

Auf den Dächern und Schornsteinen der Stadthäuser, welche dieselben Schwalbenarten wie anderswo umgaukeln, sieht man 
von Zeit zu Zeit das Hausrotschwänzchen (Lusciola titys) am ehesten noch in der Nachbarschaft der geräumigen katholischen Kirche. Seinem schönen Verwandten, dem Gartenrotschwänzchen (Lusciola phoenicurus) begegnete ich am Weidenauer Gutsgarten.

Die weiten Wiesen zu beiden Seiten der Drewenz beherrschen Wiesenpieper (Anthiss pratensis), braunkehlige Wiesenschmätzer (Pratincola rubetra) und Bachstelzen, die das Krautdickicht der Altwasser schwanzwiegend absuchen. Neben Motacilla alba findet sich auch Motacilla flava.

Die Wiesenschmätzer sind hier recht scheu und deshalb schwer genau zu beobachten. In Folge ihres freien Sitzes sehen sie den Wanderer schon von weitem ankommen und fliegen stets ab, wenn der neugierige Störenfried noch etwa 25-30 Schritt entfernt ist, sodass man selbst mit dem Opernglase wenig intime Momente aus ihrem Leben belauschen kann. An manchen Nachmittagen bin ich ihnen wohl eine kleine Weile aber die Felder nachgelaufen; immer wieder strich ich in denselben Zickzacklinien durch ihr geräumiges Revier und immer wieder erlebte ich denselben Misserfolg.

An dem Standort eines Pärchens von Motacilla flava wurde ich wiederholt Zeuge eines absonderlichen Vorgangs. Von ferne, jenseits der Häuser Neumarks, die allerdings nicht grade ein Meer bilden, erschien leichten Fluges eine männliche Kubstelze, stürzte sich auf den harmlosen Artgenossen, der beschaulich im Kraute einhertrippelte, und Hog mit ihm zankend und beissend empor, um dann eben wieder so schnurstracks, wie sie gekommen, in der blauen Ferne zu verschwinden. Ankunft, Kampf und Abzug machten einen eigentümlichen Eindruck, als läge dem Thun des fremden Ankömmlings ein strategischer Plan zu Grunde.

In den Weiden am Flusse nistet der edelste Gast dieser Fluren, der Sprosser (Lusciola philomela), der im heurigen Mai wegen der kalten Witterung seinen Gesang leider recht lange unterbrechen musste, und neben ihm erblickt man bisweilen anch einen Neuntöter (I Lanius collurio); Lanius senator fehlt dagegen.

Eher noch erblickte ich den Neuntöter in den Gesträuchen, die an den verfallenen Mauern des alten Klosters Lonk emporwachsen. Wie die Dorngrasmücke zeigte auch der Dorndreher eine grosse Abneigung dagegen, vor dem Beobachter auf die baumlose Kultursteppe zu flüchten, sondern bemühte sich, in den wenig geräumigen Sträuchern die nötige Deckung zu finden. 
Einzelne der Sprosser, die an der Drewenz stehen, bringen die F'hilipp-F'lilipp-Rufe recht schön zum Vortrag, beeinträchtigen aber den schönen Eindruck der markigen Töne gar sehr durch unschöne, ich möchte fast sagen, zerknitterte Strophen, die an Klangwirkung noch hinter dem Wettern einer Sumpfmeise zurückstehen.

In diesem Frühling hat der Sprosser hier böse Tage und Nächte durchgemacht (am 11. Mai hatten wir Nachts 3 Zoll Schnee). Am unangenehmsten schienen ihm klare Niichte mit Ostwind zu sein, wo in Folge der bedeutenden Würmeabgabe durch Struhlung die Temperatur bis auf ca. $3^{0}$ Kulte herabging. In solchen Niichten schwieg der Sprosser völlig, während ihn gewöhnlicher, gleichmässiger Landregen bei $4^{0}-5^{0}$ Wärme kaum im Gesange hinderte.

Mit dem gelben Spötter und den Grasmücken verhielt es sich grade ungekehrt. Bei $+1-2^{\circ}$ Lufttemperatur, ruhiger Luft und hellem Sonnenschein sass der gelbe Spötter unseres Schulgartens noch immer lustig auf einem sonnigen Zweige und knüpfte Strophe an Strophe, wihrend kaum fühlbarer Regen ihn sofort verstummen machte, und den Grasmficken erging es ganz ähnlich.

An sonnenhellen Maitagen mit kalter Lufttemperatur verliess die Grasmücken und Spötter sogleich ihre Lebenslust, weun die Sonne sich zum Untergang neigte und ihre schrägen Strahlen die wärmende Kraft einbüssten. Alsdann sah man stets die eben noch so fröhlichen Sänger durch's Gesträuch hüpfen.

Von Wasservögeln sieht man am ehesten noch ein Paar Enten (Anas boschas), denn die Altwasser sind nicht geräumig genug, um viele Gäste zu beherbergen. Den Strandläufern und anderen mehr fehlt der gelbe, breite, sandige Flussstrand, während den Nöwen der Fluss zu schmal, die Flut zu trübe, strudelreich und verwirrt sein dürte. Selbst die Rohrdrossel (Acrocephalus arundinaceus) findet in den Altwassern noch kein gastliches Heim; nur einen einzigen Teichsänger (Acrocephalus salicarius) sah ich im Norden der Stadt. Da ich jedoch seine klappernden Lieder schon seit mehreren Tagen nicht mehr gehört habe, muss ich annehmen, dass auch ihm unser Städtchen nicht behagt hat und er anderswo zwecks Verheiratung seine Lieder schirkt.

Das Getön der Rohrammer (Emberiza schoeniclus) schallte mir an demselben Ort recht oft entgegen. Weil das Rohr hier 
nur sehr spärlich ist, wurde der schöne Vogel zum Bewohner der Weidengebüsche, von deren Zweigen er sein Liebeslied stammelt.

An seiner Sommerresidenz steht in nächster Nähe der Stadt auch ein Pärchen Gallinula chloropus. Das sumpfige Altwasser der Drewenz, auf dem die anmutigen Teichhuhner sieleln, ist nur recht klein. Trotzdem streichen sie bei drohender Gefahr nicht ab, sondern bergen sich in den dichten Pflanzen des Sumpfes, ohne sich durch Wüfe mit Erdschollen ans ihrem Versteck auftreiben zu lassen.

Die Ansiedelung der Rohrvögel wird noch dadurch erschwert, dass die Küthner das schwankende Rohr, wo immer es sich einfand, emsiglich schneiden, um mit den langen Schäften Stall und Scheuer zu decken. Daher starren den Ankömmlingen im Lenz nur die kurzen Stoppeln entgegen und die bruitelustigen müssen weiter wandern, gastlichere Stätten zu suchen.

Sogar der Storch ist bei Neumark nicht tibermässig häufig. Sein Nest schmückt allerdings als weithin sichtbares Wahrzeichen einen zerbröckelten Wartturm der Stadtmauer, aber auch dieses Nest steht leer, und wenn der biedere Wächter der Nacht die Feuerglocke zieht, scheuchen ihre gefürchteten Töne kein Storchenkind aus dem süssen Schlummer.

Besser als für das Rohrgevögel eignen sich die Ufer der Drewenz, die bald steil abfallen, bald sanft gewölbt sind, an denen immer wieder und wieder Weidengebüsch mit weitüberhängenden Zweigen haftet, für Alcedo ispida, den fliegenden Edelstein unseres Vaterlandes. Einmal sah ich ihn pfeilschnell über das Feld fliegen und wurde von meinem Begleiter hastig auf "den Vogel ohne Schwanz mit dem dicken Bauch" aufmerksam gemacht. Im allgemeinen verlässt er jedoch nicht ohne Not sein feuchtes Brutrevier.

Ein erfreulicher Anblick ist für den ostdeutschen Ornithologen der schopftragende Wiedehopf (Upupa epops), den man bei uns in der Danziger Gegend nur recht selten zu sehen bekommt. Hier stolzierte er keck auf dem Lawn-tennis-Platz des Schulgartens umber. Allerdings erwies sich diese Vertraulichkeit des Wiesenhüppers als wenig praktisch, denn ein Schrotschuss aus der Flinte des Direktors machte seinem anrüchigen Dasein ein schnelles Ende - jetzt ziert er die Sammlung der Schule. Auch in der Umgegend des Städtchens begegnete ich dem weissgefleckten Kukuksküster zu wiederholten Malen. 
Es giebt in Westpreussen wohl kaum einen zweiten Schulgarten, der von so zahlreichen und so verschiedenen Vogelarten besucht wird, wie der Schulgarten in Neumark. Auf den Bäumen der südlichen Allee, die auf weite Felder hinansschaut, leiert die Grauammer, spinnt der Hämmerling seinen Singsang. Auf dem sandigen Lawn-tennis-Platz schreiten neben der Haubenlerche schmucke Bachstelzen einher, zu denen sich bis vor kurzem wohl gar der schmucke Wiedehopf gesellte. Wenige Schritte davon trillert der Grünfink, schlägt der Edelfink, lockt der Stieglitz mit metallischem Ruf. Dicht daneben schlüpft das Rotkehlchen schwanzwippend durch das dunkle Gezweig der ubermannshohen Fichten, die den Zaun beschatten. Im Geäst der Büsche und Bäume singen Zaun- und Dorngrasmücken und der Spötter, der Töne reichster, während Sumpf- und Kohlmeisen emsiglich jedes Ästchen und jedes Astloch nach ihren mikroskopisch kleinen Nahrungsteilchen absuchen. Ihre feinen Stimmchen werden allerdings gar leicht von den blöden Spatzen überschrieen, die hier wie anderswo das letzte Wort behalten.

Die groben Schreier geniessen im Schulgarten Bürgerrecht, weil man seitens des Lehrerkollegiums ihre Verdienste im Vertilgen der Maikäfer, die hier bisweilen zur tiberaus lästigen Landplage werden, gebuhrlich, vielleicht uber Gebuhr preist. Jedenfalls scheinen die Spatzen hier der Ansiedlung anderer Vögel nur wenig zu schaden, da sie sich in dem geräumigen Hof- und Gartenraum auf wenige Lieblingsplätze in der Nähe der Gebäude beschränken und den übrigen Platz dem anderen Gefieder kampflos überlassen.

Im Herzen des Ornithologen regt sich beim Anblick dieses prächtigen Schulgartens der stille Wunsch, alle Anstalten möchten es so gut haben, dann würde der Nachwuchs unserer Zunft auch vielleicht nicht gar so spärlich sein.

Durch regelmässige Beiträge aus kargem Taschengeld sind die Schüler am Gedeihen der Anlagen interessiert, direktoriale Verfehmungen etwaiger Nestzerstörer thun ein weiteres, und so leben denn hi'r die zerstörungslustigen Menschenkinder und die schutzbedürftige Brut der Vögel einträchtlich nebeneinander, wie es sein kann und sein soll.

Der Wald von Kosten, der östlich von Brattian ins Drewenzthal hinabsteigt, ist an Vögeln nicht arm. Im Unterholz nistet überall das Rotkehlchen (Lusciola rubecula) und aus den Baum- 
kronen tönt uns das wohlbekannte Zilp-Zalp des Weidenlaubvogels (Ficedula acredula) entgegen. Neben dem Finken (Fringilla coelebs) hüpft geräuschlos der Fitis- und Schwirrlaubvogel (Ficedula trochilus und $F$. sibilutrix) durchs hohe Buchenstangenhole und kreischend fliegt der Eichelhäher (Garrulus glandarius) vor uns auf. In sonniger Lichtung singen die Grasmücken und auch die verhallenden Töne des Baumpiepers (Anthus arboreus) dringen an unser empfängliches $\mathrm{Ohr}$. Die Drosselarten sind dieselben wie sonst in der Provinz, iber Turdus pilaris vermochte ich wenig in Erfahrung zu bringen. Die Förster behaupteten, sic niste in diesen Wäldern nie, doch pflege ich, durch Schaden gewitzigt, auf solche Aussagen nur wenig zu geben. An engbebuschtem Örtchen fehlt auch der Zaunkönig (Troglodytes parruluss) nicht. Einer der kleinen Schelme musste an einem Hohlweg, wo lockerer Sand unter der überhängenden Rasendecke fortgeglitten war, uber schier metertiefe Schlupfwinkel verfügen, denn selbst der tastende Stock vermochte ihn nicht aus seiner Festung zu verscheuchen.

Von Raubzeug sollen Sperber (Astur nisus) hier häufiger sein als in der Nachbarschaft, wenigstens versicherte mir der Förster in Kaszek, dass er allsommerlich mehrere dieser Räuber erlegt. Im allgemeinen sind aber meine Erfahrungen und Kenntnisse des hiesigen Raubzeuges so lückenhaft geblieben, dass ich mit denselben besser nicht aufwarte.

Damit wäre die hiesige Ornis in grossen Zügen geschildert, wie sie das Drewenzthal um Neumark herum, von Brattian bis Kaluga, besiedelt. Vergleichen wir diese Ornis mit der des Danziger Weichbildes, so fällt uns besonders auf.

1. Das Fehlen von Fringilla serinus.

2. Das fast gänzliche Fehlen von a) Sturnus vulgaris,

b) Corvus pica.

3. Das verhältnismässig häufige Vorkommen von Opupa epops.

4. Die ungemein grosse Häufigkeit von Emberiaa miliaria und ihr vertrautes Zusammenleben mit den andern Ammerarten.

Das sind verhältnismässig bescheidene Resultate. Wenn man aber bedenkt, dass manche Meile durchwandert wurde, und manche Stunde auf den feuchten Wiesen mir eintōnig dahinschlich, ehe sich ein mildherziger Vogel des Beobachters erbarmte, dann 
wird man dieses bescheidene Resultat vielleicht nicht ganz verachten. Wenn aus dem mit Ornithologen reicher gesegneten Westen nur gediegenes Gold Gnade findet vor dem Auge des Lesers, sollte man aus unserm armen Osten auch das grünspanige Kupfer nicht verachten.

Neumark, 22. V. 1900.

\section{Eine weissliche Farbenvarietät der Marzente, Areas boschas $\mathrm{I}$. \\ Von C. Wüstnei.}

(Mit Abbildung).

Spielarten und Farbenvarietäten der wilden Enten im allgemeinen, sowie der Märzente im Besonderen, gehören bekanntlich zu den grössten Seltenheiten, sodass selbst Naumann kaum etwas von solchen Abarten zu berichten weiss, er sagt, dass man, abgesehen von den mit Hausenten gekreuzten Bastarden, unter vielen Hunderten noch nicht eine mit einer ungewöhnlich gefärbten Feder findet. Er spricht dann von einem auf weissem Grunde isabellfarbig gefleckten Weibchen, welches er selbst besass und von einer schwärzlichen Varietät eines Männchens im Hochzeitskleide, welches im Brandenburgischen geschossen sein soll und in Frisch's Vögel als Anas boschas nigra auf Tafel 193 abgebildet wurde. Das ist Alles, was Naumann weiss, und auch mir war bisher anderweit von derartigen Spielarten nichts bekannt geworden, ebenso wie mir seit vielen Jahren unter den auf den Mecklenburgischen Seen beobachteten und erlegt gesehenen Wildenten niemals eine auffallende Erscheinung vorgekommen war, bis im Herbst vorigen Jahres eine helle Varietät einer männlichen Märzente in hiesiger Umgegend beobachtet wurde, welche mit einer grösseren Schar anderer Märzenten uberwinterte und hier von mir recht oft gesehen wurde. Dieser Vogel wurde dann in den letzten Tagen des Februar 1901 auf dem südlichen Teil des Schweriner Sees erlegt, gelangte in den Besitz des Präparator Knuth, welcher ihn ausstopfte.

Diese Varietät lässt nicht etwa den Gedanken an eine Verbastardierung mit einer weissen Hausente aufkommen, die schlanke Gestalt, der ganze Habitus, die Grössenverhältnisse aller Teile, ebenso das ganze scheue Wesen sprechen unzweifelhaft für eine reine Wildente. Wie gewöhnlich versammelten sich auch im 